\title{
Percepciones docentes en torno a los procesos de institucionalización del aprendizaje-servicio en educación superior: un estudio de caso
}

\author{
Rocío Almendras-Chacana \\ Alicia Pérez-Lorca \\ Universidad de Santiago de Chile, Chile
}

\section{Resumen}

La metodología de aprendizaje-servicio ha sido altamente valorada en educación superior gracias a las potencialidades que tiene para el desarrollo de habilidades diversas y complejas, altamente necesarias para la inserción efectiva y oportuna de los profesionales que el mundo de hoy requiere. Dentro de sus características fundamentales destaca la posibilidad de que el estudiantado conozca las necesidades de la sociedad en la que vive, mediante la vinculación directa con comunidades con problemáticas sentidas y manifiestas. Con ello, introducir innovaciones en educación superior se convierte en una tarea compleja, muchas veces movilizada por intereses de los mismos docentes y con dificultades para su formalización dentro de la institución. Aun cuando existe literatura orientadora respecto de cómo implementar procesos de institucionalización, las voces de sus principales actores no son comúnmente recogidas. El propósito de este estudio es analizar las percepciones de un grupo de profesores que implementan aprendizaje-servicio en una universidad chilena de carácter estatal.

\section{Palabras clave}

Aprendizaje-servicio, institucionalización, profesorado universitario, innovación educativa.

Fecha de recepción: 10/III/2019

Fecha de aceptación: 10/V/2019

Almendras-Chacana, R. y Pérez-Lorca, A. (2019). Percepciones docentes en torno a los procesos de institucionalización del aprendizaje-servicio en educación superior: un estudio de caso. RIDAS, Revista Iberoamericana de Aprendizaje Servicio, 7, 17-36. DOI10.1344/RIDAS2019.7.2 


\title{
Teacher perceptions about the institutionalization of Service-Learning in Higher Education: a case study
}

\begin{abstract}
The service-learning methodology has been highly valued in higher education thanks to its potential for the development of diverse and complex skills, which are essential for the students' effective and timely insertion into professional life. Among the many benefits that service-learning brings to students is the possibility it affords them to learn about the needs of the society in which they live, by creating direct links with communities with perceived and manifest problems. Despite the above, introducing such innovations in higher education can still be a complex task, which usually faces many obstacles to become formally recognized by institutions and is mainly achieved thanks to academic staff's determination in pursuing their interests. Although there is guidance literature on how to successfully achieve institutionalization, the main actors' voices are not usually represented. The purpose of this study is to analyse the perceptions of a group of teachers who implement service-learning at a public Chilean university.
\end{abstract}

\section{Keywords}

Service-learning, institutionalization, university teaching staff, educational innovation. 


\section{Introducción}

El año 2015 la comunidad internacional se sumió en una profunda discusión acerca del futuro de la humanidad. Su expresión fue la creación de la Agenda 2030 para el Desarrollo Sostenible (ONU, 2015), materializada en la definición de 17 Objetivos de Desarrollo Sostenible (en adelante ODS) cuya finalidad es "garantizar una vida sostenible, pacífica, próspera y justa en la tierra para todos, ahora y en el futuro" (UNESCO, 2017, p.11). Para ello, la educación se convierte en un motor crucial, en la medida que, además de garantizar la adquisición de competencias básicas y habilidades cognitivas, interpersonales y sociales; permita también

(...) el desarrollo de las competencias, los valores y las actitudes que permiten a los ciudadanos llevar vidas saludables y plenas, tomar decisiones con conocimiento de causa y responder a los desafíos locales y mundiales mediante la educación para el desarrollo sostenible y la educación para la ciudadanía mundial. (UNESCO, 2015, p.8).

Formar competencias implica un gran reto, pues "no se pueden enseñar, sino que los mismos alumnos deben desarrollarlas. De este modo se adquieren durante la acción, sobre la base de la experiencia y la reflexión" (UNESCO, 2017, p.7). A su vez que para convertirse en ciudadano es necesario conocer y comprender la sociedad en la que vivimos que, desde un ejercicio profesional, suponga la contribución a un mundo más justo y próspero.

En este contexto, y desde una perspectiva de los procesos de enseñanza y aprendizaje, la metodología de aprendizaje-servicio representa una innovación educativa de gran valor, pues permite desarrollar tanto competencias del dominio disciplinar, como aquellas vinculadas a aspectos valóricos, vocacionales y sociales, entre otros (Puig, Gijón, Martín y Rubio, 2011).

Por otra parte, y desde una mirada más institucional, el aprendizaje-servicio se configura como una estrategia de gran impacto para potenciar la relación de la academia con la comunidad, la vinculación de su estudiantado con las necesidades de su entorno más próximo (Bringle y Hatcher, 2000; Santos, Sotelino y Lorenzo, 2015).

A continuación, se presenta un estudio que tiene por objetivo analizar la visión de los docentes en torno a los procesos de institucionalización desarrollados en una institución de educación superior estatal chilena.

\section{Marco teórico y referencial}

2.1. La metodología de aprendizajeservicio como promotora de aprendizajes de calidad

Algunos estudios sitúan el origen del aprendizaje-servicio en las estrategias de servicio comunitario implementadas en las universidades de Estados Unidos en la década de 1960 (Santos et al., 2015). Desde esa fecha a la actualidad, se han desprendido una serie de 
estrategias con la misma raíz, pero con algunas características diferenciadoras.

Para efectos de este estudio, comprendemos el aprendizaje-servicio como un programa pedagógico que articula intencionadamente una serie de contenidos disciplinares de distinta naturaleza, con acciones de servicio solidario destinado a atender las necesidades sentidas de una comunidad (Tapia, 2016). En este sentido, se releva la importancia que tiene poner en acción el saber académico con las demandas que un colectivo particular pueda tener y que dicho saber pueda ayudar a resolver o despejar.

Del mismo modo, la participación activa de los estudiantes es un componente fundamental de la metodología (Tapia, 2016), la cual puede expresarse en la búsqueda y selección de comunidades, en el levantamiento de problemáticas conjuntamente, en el diseño de proyectos, la implementación de iniciativas y la evaluación de las mismas. Las actividades que para ello se ejecuten, requerirán garantizar espacios para la reflexión, elemento central de la metodología (Páez y Puig, 2013).

Como toda metodología de enseñanza y aprendizaje de naturaleza compleja, el aprendizaje-servicio está compuesto por una serie de estrategias y técnicas que permiten desarrollar distintos tipos de habilidades, como por ejemplo, el análisis de información, elaboración de proyectos, toma de decisiones y trabajo en equipo, por nombrar algunas. Por ello, muchas veces es confundida con otras metodologías y estrategias de aprendizaje activo y experiencial, como el método de proyectos (Geruzaga y García, 2018).

Sin embargo, el aprendizaje-servicio tiene una fuerte intencionalidad social (Tapia, 2010; Martínez, 2010), la que es fácilmente reconocible porque, además de desarrollarse en un contexto real como podría ser el método de proyectos, enriquece el aprendizaje de los futuros profesionales y el devenir de la comunidad en la que se inserta gracias al fin social-transformador que posee (Martínez, Medero, Ibáñez y Sánchez, 2018).

Ahora bien, como ya señalamos, existen otras estrategias que permiten formar habilidades de la misma naturaleza que el aprendizaje-servicio. Sin embargo, el aprendizaje-servicio posee dos características diferenciadoras que vale la pena señalar (Tapia, 2010). En primer lugar, el trabajo con las comunidades obliga a hacerse sensible a las necesidades de la sociedad, pues los proyectos deben ser elaborados conjuntamente, no solo a partir de lo que los estudiantes puedan ofrecer como futuros profesionales. Esta acción implica muchas veces aprender a ser receptivo, dialogante y conciliador, porque el beneficiario del servicio es un grupo humano real, no imaginado como ocurre en ocasiones con el aprendizaje basado en proyectos.

En segundo lugar, acercarse a las necesidades de la sociedad desde una mirada como profesional compromete al estudiante en su rol dentro del mundo en el cual vive. La responsabilidad social que implica sentirse parte, pero también activo miembro de la sociedad, le permite al 
estudiantado proyectar su acción profesional en la línea que nos propone la UNESCO en la Agenda 2030 expresada en los ODS. A su vez, la comprensión de esta nueva forma de convivir, permite el desarrollo de competencias de formación ciudadana que, hasta el momento, difícilmente es abordado en el contexto de educación superior, incluso cuando forma parte de los perfiles de egresos de muchas instituciones educativas.

Tal ha sido el interés por el aprendizajeservicio que desde el año 2000 ha crecido el interés por difundir las buenas prácticas mediante la generación de redes y conferencias agrupando iniciativas de este tipo (Martínez, Martínez, Alonso y Gezuraga, 2013). Para el año 2002, se funda el Centro Latinoamericano de Aprendizaje y Servicio Solidario -CLAYSS- y en el año 2008 nace en EEUU la International Association Research on ServiceLearning and Community Engagement IARSLCE-. Más adelante comienzan a formalizarse las redes al interior de cada país como fue en 2010 la creación de la Red Española de AprendizajeServicio y la red chilena REASE en 2011 (Gezuraga y Herrero, 2017; Santos, et. al, 2015 ). Con el afán de estrechar lazos entre los participantes y formalizar las iniciativas existentes, distintas universidades iberoamericanas han consolidado de grupos de innovación en aprendizaje-servicio movilizados por los mismos académicos. Lo anterior, sumado a la necesidad de visibilizar las iniciativas y conseguir nuevos recursos, ha dado como resultado un importante proceso de institucionalización.

\subsection{Los procesos de institucionalización}

Seguramente el lector ha sido testigo de cómo exitosos proyectos de aprendizaje-servicio desaparecen en el tiempo ya sea por falta de recursos y poco apoyo de las propias facultades, así como por deficientes sistemas de comunicación con los socios comunitarios y recambio permanente del cuerpo docente. Es por ello, que los cambios serán sostenibles solo, si junto a la innovación, se avanza en la institucionalización (Martínez et al., 2013).

Desde una perspectiva organizacional, la institucionalización sería la fase final de un cambio (Jouannet, Ponce, Montalva y Von Borries, 2015). Sin embargo, desde una mirada educativa, la institucionalización no sería un estadio final, sino más bien un estado continuo, donde la posibilidad de incorporar de manera permanente algunos cambios que han implicado mejoras para los objetivos de la entidad, sería una forma de "convertir dichos cambios en hábitos docentes colectivos que ya no dependan de la motivación, la iniciativa, la voluntariedad de una persona o grupo de personas, sino que formen parte de una identidad pedagógica de una institución" (Malpica, 2013, p. 208).

Para Furco (1999), la institucionalización debe considerar el paso a través de tres fases: 1) la creación de masa crítica al interior de la organización; 2) la construcción de calidad de las iniciativas; y 3 ) la sustentabilidad institucional.

De esta manera, sería posible transitar

Almendras-Chacana, R. y Pérez-Lorca, A. (2019). Percepciones docentes en torno a los procesos de institucionalización del aprendizaje-servicio en educación superior: un estudio de caso. RIDAS, Revista Iberoamericana de Aprendizaje Servicio, 7, 17-36. DOI10.1344/RIDAS2019.7.2 
desde un interés inicial por la metodología hacia su implementación sustentable en el tiempo, que no dependa del compromiso e interés individual o de un grupo reducido de personas, sino de un esfuerzo de toda la organización.

A su vez, autores como el mismo Furco (2001) y Bringle y Hatcher (2000) coinciden en que la institucionalización del aprendizaje-servicio tiene mejores resultados si incorpora una serie de elementos tales como: soporte económico, administrativo y material de las iniciativas, reconocimiento institucional de las actividades de aprendizaje-servicio y alineamiento con las metas institucionales. Iremos desarrollando estas ideas a continuación.

\section{a. Reconocimiento institucional}

Este tipo de formalización obedece a la declaración que realiza la institución respecto de la importancia del aprendizaje-servicio dentro de la organización. Existen diversas formas de realizar este reconocimiento, ya sea mediante los grandes lineamientos formativos y estratégicos, como mediante la asignación dirigida de recursos (Furco, 2001; Bringle y Hatcher, 1996)

En el caso de la asignación de financiamiento especial para las iniciativas de aprendizaje-servicio, ya sea como proyectos concursables o bien como asignación específica de recursos a las unidades académicas, su visibilidad es bastante clara para la comunidad. Sin embargo, el reconocimiento institucional va mucho más allá.

En el contexto académico, la promoción de las iniciativas de aprendizaje-servicio puede provenir de la creación de material de divulgación mediante plataformas y publicaciones permite visibilizar los proyectos (Bringle y Hatcher, 2000). Del mismo modo, la creación de incentivos (en dinero u honorarios) a los profesores que impulsan las innovaciones permite demostrar que este tipo de iniciativas son parte de las actividades académicas de la universidad (Santos et al., 2015).

Sin embargo, vale la pena tener en consideración que en un estudio realizado por Bell y otros (2000, citado en Furco, 2001) que recopiló información de 45 universidades norteamericanas, fue posible observar que incluso cuando existían incentivos institucionales, muchos académicos aceptaban destinar tiempo y energía a implementar un proyecto de aprendizaje-servicio solo cuando estaban convencidos que ese compromiso no sería visto negativamente por sus pares o jefaturas. Es decir, el reconocimiento institucional no obedece solamente a la entrega de estímulos aislados, sino que debe configurarse articuladamente con los procesos de jerarquización académica, de manera de no resultar contradictorio con la política institucional.

Lo anterior se vincula fuertemente con un cambio cultural, que permita otorgar cada vez más reconocimiento a las actividades docentes dentro de la universidad al mismo nivel de la investigación. En esta línea, Furco 
(2001) señala que existe una desproporcionada atención a las actividades investigativas como la publicación de artículos y proyectos de investigación, en desmedro de las actividades vinculadas a la docencia. En los contextos de universidad tradicionales, los académicos nóveles están fuertemente tensionados por responder a estos indicadores de producción científica, lo que hace difícil que se involucren en proyectos de aprendizaje-servicio como se señaló en el párrafo anterior.

En este sentido, el mismo Furco (2001) propone para las universidades centradas en la investigación, poner atención en tres elementos esenciales: 1) el aprendizaje-servicio debiese vincularse más estrechamente a las actividades de mayor valor dentro de los académicos; 2) el aprendizajeservicio debiese alinearse a las metas académicas más importantes; y 3) el aprendizaje-servicio debiera incorporarse estratégicamente en la estructura de la universidad.

Así, el aprendizaje-servicio se transforma en una manera de materializar el proyecto de una institución educativa, mediante la creación de una masa crítica que valide a su vez la actividad docente como una más de las actividades académicas propias de la universidad (Santos et al., 2015).

De esta forma, el aprendizaje-servicio podrá ir más allá de la ejecución de proyectos (Martínez et. al, 2013) y podrá incentivarse la actividad investigativa en relación al estudio de las experiencias realizadas, al igual que otros fenómenos.

\section{b. Integración curricular}

La integración curricular sería una de las maneras más concretas de formalizar el aprendizaje-servicio en el interior de una institución educativa (Jouannet et al., 2015; MartínezUsarralde, Zayas, y Sahuquillo, 2016; Puig et al., 2011; Stater y Fotheringham, 2009; Tapia, 2016).

En un nivel superior de integración curricular se encontrarían las declaraciones institucionales contenidas en los marcos generales de cada institución, como puede ser el modelo o proyecto educativo institucional (Tapia, 2016; Phillips, Bolduc, y Gallo, 2013).Si bien no se trata de acciones particulares ni concretas, su valor radica en el poder político (Carr, 1996) que tienen dichas declaraciones, en la medida que conforman una hoja de ruta respecto de cómo alinear el aprendizaje-servicio con los propósitos institucionales.

Es por ello que para autores como Laura Campo (2015), la institucionalización del aprendizajeservicio se trataría de una opción ideológica y política, reconociendo que el rol de la educación es transformar la sociedad en una de tipo más equitativa. El aprendizaje-servicio tendría sentido en universidades socialmente comprometidas, fuertemente convencidas en que su misión es formar ciudadanos. Si una universidad se define como socialmente responsable, no tendrá problema en promover el aprendizaje-servicio dentro de la institución como una forma de 


\section{concretar sus metas.}

Desde un nivel curricular medio, es decir a nivel de planes de estudio, Nieves Tapia (2016) reconoce fundamentalmente cinco maneras de integrar el aprendizaje-servicio en el currículum, como 1) una actividad obligatoria o voluntaria al interior de una asignatura, a modo de estrategia didáctica; 2) una asignatura completa, obligatoria u optativa, de un plan de estudios, a modo de metodología; 3) una modalidad de práctica profesional de una carrera; 4) una modalidad de realizar tesinas o trabajos de grado y postgrado, como base de un proyecto de investigación; y 5) dentro de un proyecto de extensión o voluntariado, ya sea a partir de las oficinas de extensión o en vinculación explícita a las cátedras con las cuales puedan vincularse.

En una línea similar, autores como Stater y Fotheringham (2009), consideran la integración curricular del aprendizaje-servicio desde su formalización en los planes de estudio, pero más bien desde una perspectiva flexible, a partir de la incorporación de un número determinado de créditos a los requisitos de titulación.

Sobre ello, algunos autores (Ruiz, Meneses y Montenegro, 2014) invitan a considerar que la implementación de experiencias de aprendizaje-servicio de manera informal y no bajo los instrumentos curriculares de nivel medio, podría alterar la coherencia del plan de estudios.

Así mismo, las problemáticas en la coherencia curricular también impactarían a nivel didáctico, pues no explicitar el aprendizaje-servicio en el programa de la asignatura o bien no articular sus resultados de aprendizaje con las acciones evaluativas, podría desafiar el alineamiento constructivo de la misma (Biggs, 2006).

De todos modos, considerando que el aprendizaje-servicio es una metodología que requiere de alto compromiso y participación del estudiantado, existen autores que cuestionan su condición de obligatoriedad en el currículum, pues sería entendida "una exigencia burocrática, antes que como factor de calidad en la educación de los jóvenes" (Santos et al., 2015, p. 73).

Ahora bien, la formalización curricular no es suficiente para hacer del aprendizaje-servicio una práctica sistemática, permanente, esperada y legitimada (Gezuraga y Herrero, 2017).

\section{c. Soporte institucional}

El soporte institucional podría ser considerado cualquier tipo de apoyo que la institución facilite a los profesores para llevar a cabo la implementación de una metodología de enseñanza y aprendizaje en aprendizaje-servicio. En este caso lo abordaremos desde la perspectiva de aquellos recursos humanos, materiales o financieros que permitirán llevar a cabo un programa de aprendizajeservicio y mantenerlo en el tiempo (Tapia, 2010; Bringle y Hatcher, 2000)

A nivel de recursos humanos, sin duda uno de los elementos primordiales es formar pedagógicamente a los docentes

Almendras-Chacana, R. y Pérez-Lorca, A. (2019). Percepciones docentes en torno a los procesos de institucionalización del aprendizaje-servicio en educación superior: un estudio de caso. RIDAS, Revista Iberoamericana de Aprendizaje Servicio, 7, 17-36. DOI10.1344/RIDAS2019.7.2 
en la metodología de aprendizajeservicio: su propósito, etapas, actores relevantes, hitos, procedimientos y mecanismos de evaluación. Del mismo modo, será necesario, preparar tanto al profesorado como a los asesores, en estrategias para la vinculación con los socios comunitarios y formas de introducir las iniciativas en el currículum de sus carreras. Lo anterior puede implicar disponer de recursos para dictar cursos, seminarios y talleres, así como contratar asistencias externas para formar a los responsables (Bringle y Hatcher, 1996 y 2000).

En este mismo sentido, la formalización debiese implicar la creación de estructuras institucionales que brinden soporte -tanto pedagógico como administrativo-, así como que resguarden el monitoreo de dichas iniciativas (Stater y Fotheringham, 2009). De alguna manera, debiesen garantizarse los esfuerzos para "situar la actividad en un espacio curricular del horario, disponer de instancias de programación y evaluación, institucionalizar la figura del referente de los proyectos y disponer de algunos recursos y organizarlos debidamente para la realización de la experiencia innovadora" (Puig, et.al, 2011, p.60).

Para evitar la multiplicación de esfuerzos, autores como Bringle y Hatcher (2000) señalan la importancia de establecer una oficina dependiente de la dirección académica, que brinde asistencia de tipo técnica, logística y monetaria. Que dicha oficina sea la encargada de centralizar la planificación estratégica, los recursos asignados, los reconocimientos e incluso la búsqueda de nuevos académicos participantes.
Del mismo modo, debiera también generar las pautas para la adecuada destinación de tiempo y las discusiones que se espera guíen estas iniciativas. Lo anterior, permitiría asumir las diversas tareas que se despliegan antes la implementación de experiencias de Aps, entre las que destacan, entre otras (Bringle y Hatcher, 1996):

- desarrollo de procedimientos de sistematización (cuantificación de experiencias, carreras y asignaturas involucradas, estudiantes y docentes involucrados)

- definición de un plan de apoyo financiero que asegure la sustentabilidad de las iniciativas

- acciones de monitoreo (elaboración de reportes anuales, implementación de evaluaciones de satisfacción)

- gestión de la vinculación con los socios comunitarios (catastro, búsqueda de socios comunitarios),

- generación de equipos de investigación, además de la difusión intra e interinstitucional (participación en redes de aprendizaje-servicio y seminarios).

En este contexto es importante recordar que los socios comunitarios forman parte trascendental en el diseño e implementación del aprendizajeservicio. A nivel de soporte institucional será necesario resguardar apoyos, no tan solo para la elaboración de convenios, sino también para clarificar las expectativas de todos los actores en el proceso, mediante estandarización de protocolos, cartas de compromiso, 


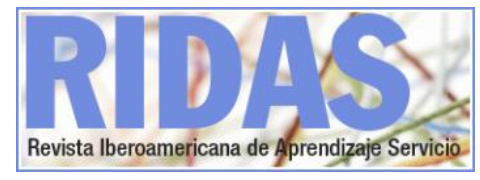

autonomía de acción y un trato igualitario entre ambas partes (Stater y Fotheringham, 2009). De esta forma, mientras más serios sean los proyectos de aprendizaje-servicio en la universidad, mayor compromiso existirá con los socios y, con ello, mejores resultados tendrán dichas experiencias.

Junto a lo anterior, Agrafojo, GarcíaAntelo y Jato (2017) insisten en la importancia que todo proceso de institucionalización considere tanto el acompañamiento y asesoría, como la evaluación de los proyectos implementados, con ello favoreciendo a los procesos de formación y mejora. Esto se insertaría en la perspectiva que la innovación educativa es un proceso continuo de cambio y mejora (Romero, 2003; Mauri, Coll C.y Onrubia, 2007).

Bajo la lógica de la evaluación de procesos e iniciativas, Furco (2011) propuso una rúbrica para autoevaluar institucionalmente el grado o nivel de formalización de las iniciativas de aprendizaje-servicio. Las dimensiones consideradas para evaluar el trayecto de la institución fueron a) Filosofía y misión del aprendizaje-servicio; b) Involucramiento y apoyo de los docentes; c) Involucramiento y apoyo de los estudiantes; d) Participación y asociación de los socios comunitarios; y e) Apoyo institucional. En ellas se observar la importancia que otorga el autor a la participación de todos los actores del aprendizaje-servicio, tanto profesores, como estudiantes y socios comunitarios.

Finalmente, a nivel general, es importante señalar que la institucionalización de cualquier iniciativa debe considerar la manera en que se decide implementar el currículum. Existen experiencias de rápida institucionalización de experiencias de innovación mediante modelos top-down, que resultan de la imposición de estándares y modalidades de trabajo por los altos puestos directivos, pero que tienen un bajo nivel de empoderamiento en los docentes y estudiantes. Para evitar dichos obstáculos, y en la perspectiva de un modelo de tipo bottom-up, autores como Agrafojo et al. (2017) señalan la relevancia de hacer sinergias entre las distintas iniciativas de aprendizaje-servicio tanto dentro de la universidad como fuera de ella. Eso podría implicar estrechar la relación entre los académicos con iniciativas de este tipo, así como privilegiar el diálogo entre unidades u oficinas con tareas que pudiesen ser convergentes para esta metodología, pero que no necesariamente trabajan en conjunto, como formación del profesorado y responsabilidad social universitaria.

Con todo ello, Zlotkowski (en Furco, 2011) nos llama a no olvidar que cualquier intento de institucionalización del aprendizaje-servicio (o de cualquier otro esfuerzo de reforma) en la Educación Superior toma tiempo, compromiso y persistencia.

\section{El contexto de nuestro estudio}

El marco de este estudio se desarrolla en una universidad estatal, de larga tradición en Chile, cuyos valores institucionales están fuertemente enraizados en la excelencia, la libertad de pensamiento y expresión, el respeto a las personas, la diversidad y 
pluralismo, la cooperación y la inclusión y responsabilidad social. De esta forma, el sello de sus estudiantes está marcado por el trabajo en equipo, el liderazgo, la adaptabilidad, la postura ética en su desempeño profesional y la responsabilidad social y conciencia ciudadana, de acuerdo a su proyecto educativo.

Es una institución caracterizada por la inclusión social, por lo que ha realizado y liderado diversas iniciativas que amplían y diversifican sus mecanismos de ingreso los últimos 20 años. Ello ha derivado en que la composición del alumnado (22.800 estudiantes), es representativa de los diversos sectores de la sociedad.

En el contexto del aprendizaje-servicio, esta institución registra sus primeras iniciativas en 2005, lo que motivó inicialmente la autogestión de sus docentes por la formación en la metodología, en un gran seminario interuniversitario en 2010. El mismo año fue creada una unidad de mejoramiento de la docencia, cuya finalidad es apoyar todas las iniciativas de innovación en la universidad, incluidas las de aprendizaje-servicio.

Con la mención del aprendizaje-servicio en los grandes lineamientos institucionales de 2013, se intensificaron las acciones de perfeccionamiento, concretizados en cursos -en modalidad presencial y online-, talleres y acompañamiento directo. Algunas de dichas actividades fueron dictadas por especialistas internacionales de gran experiencia en la temática. Todo esto ha significado la participación aproximada de 88 docentes desde 2013 a la fecha de este estudio.

Considerando que esta institución posee casi una década de esfuerzos en la implementación de iniciativas de aprendizaje-servicio, pero que incluso contando con apoyos a nivel institucional la evolución ha sido variable; nos preguntamos: ¿por qué el número de iniciativas en aprendizajeservicio no ha crecido en el tiempo? ¿cuáles son los facilitadores, pero sobre todo los obstáculos que los académicos reconocen como fundamentales? Para ello, hemos recurrido a la voz de académicos con larga tradición en la implementación de aprendizajeservicio. A continuación, presentaremos las decisiones metodológicas tomadas para el desarrollo de este estudio.

De este modo, el estudio cobra relevancia en relación a las posibilidades para la institución (en tanto nos permitirá contar con información fundamentada y actualizada que nos posibilite la toma de decisiones para fortalecer el desarrollo del aprendizaje-servicio en la institución), además contribuir de forma explícita a la teorización e investigación de los procesos de institucionalización del aprendizaje-servicio.

\section{Metodología}

Considerando el valor de la investigación en la mejora de los procesos educativos desde una perspectiva sociocrítica (Coll, 2001; Coll, Colomina, Onrubia y Rochera, 2001) se abordó una investigación con método de estudio de caso (Stake, 1998; Marchant y Foster, 2017) en una

Almendras-Chacana, R. y Pérez-Lorca, A. (2019). Percepciones docentes en torno a los procesos de institucionalización del aprendizaje-servicio en educación superior: un estudio de caso. RIDAS, Revista Iberoamericana de Aprendizaje Servicio, 7, 17-36. DOI10.1344/RIDAS2019.7.2 
universidad estatal tradicional chilena. El caso corresponde a un caso de tipo único e intrínseco, pues permitirá levantar información para el análisis y mejora del objeto de estudio (Stake, 1998). En ese sentido, se realizó un diseño de carácter exploratorio (Hernández, Fernández y Baptista, 2010; Canales, 2006), en la medida que no existen estudios que analicen el estado de los procesos de institucionalización en el contexto de la institución estudiada.

Considerando que la literatura ya revela cuales son algunos de los obstáculos para la institucionalización del aprendizaje-servicio a nivel general, creemos importante estudiar en voz de los profesores cuál es su evaluación de la situación en el contexto de la institución, en la medida que puedan darnos orientaciones más precisas respecto de donde enfocar los esfuerzos para la mejora. La población considerada en este estudio fueron siete docentes vinculados a proyectos de aprendizaje-servicio. Los criterios de inclusión consideraron constituir un grupo diverso en cuanto a género y edad, así como años de experiencia implementado aprendizaje-servicio.

Los datos fueron recogidos mediante un grupo focal, por considerar que esta técnica permitía promover la discusión grupal como una manera de comprender más profundamente las experiencias, vivencias y creencias de los participantes (Mella, 2010). Ello nos permitió advertir las visiones y construcción de significados de los docentes sobre los procesos analizados (Canales, 2006; Pérez, 2007). El grupo focal se realizó sobre la base de preguntas semiestructuradas tomando como referencia las cinco dimensiones de análisis de los procesos de institucionalización propuestas por Furco (2011) expuestas anteriormente. Se decidió por utilizar esta guía por considerar que permitirían centrar la discusión sobre aspectos fundamentales de la institucionalización según la literatura especializada. La pauta de preguntas del grupo fue sometida a juicio de tres expertos del área de la educación.

Luego de la aplicación de los instrumentos, se realizó un análisis de contenido sobre la información obtenida del grupo focal, dando sentido a la información recogida, buscando categorías relevantes para el análisis, descubriendo el significado de los conceptos (Rodríguez y Valldeoriola, 2009). Se utilizaron como categorías iniciales las cinco dimensiones de la rúbrica de autoevaluación del aprendizaje-servicio, pero al considerar que los temas presentes en la discusión fueron más precisos, se redefinieron para facilitar el análisis (Pérez, 2007).

La matriz de categorización fue puesta a prueba una vez por ambas investigadoras en el $100 \%$ de los datos. Al contrastar los resultados, se realizaron ajustes pertinentes a la matriz de categorización y se procedió a recodificar conjuntamente los datos del grupo focal, dando como resultado el análisis que sigue.

\section{Resultados}

Del análisis de la información obtenida del grupo focal de docentes emergen una serie de categorías que se 
constituyen en ideas fuerza fundamentales para el análisis de los procesos de institucionalización en la universidad. Estas categorías se relacionan con las ideas sobre aprendizaje-servicio, reconocimiento institucional, integración curricular y soporte institucional.

\section{A. Ideas sobre el aprendizaje-servicio}

Los docentes participantes caracterizan el aprendizaje-servicio como una metodología educativa, de carácter participativa y activa, que permite descubrir problemáticas en su campo de ejercicio profesional. Resulta curioso que solo uno de los participantes reconoce la impronta social de esta metodología, como se observa a continuación:

"Es poner una problemática y a partir de esa problemática el alumno sea capaz de descubrir, una problemática social, que se enmarca dentro del ramo" . (Grupo focal, 17 de agosto 2016)

Sin embargo, es mucho más generalizada la valoración de aprender haciendo que esta metodología tiene, probablemente por la posibilidad que supone experimentar mediante el descubrimiento en la acción, como se observa en las siguientes citas:

"Es una forma más natural de aprender, que es como todos aprendimos. Todo el conocimiento cultural que nosotros tenemos, lo tenemos mediante ensayo y error, mediante el descubrir problemáticas. Entonces es un recurso elástico, flexible, es una metodología interesante para la educación en general". (Grupo focal, 17 de agosto 2016)
"Aprendizaje-servicio nos permite que ellos [los estudiantes] se enfrenten a un problema real, sin ninguna herramienta al principio y que esas herramientas después las vayan descubriendo y vayan viendo que esas herramientas son útiles". (Grupo focal, 17 de agosto 2016)

Resulta claro que el centro del aprendizaje-servicio es relacionado con la resolución de problemáticas. Como habilidad cognitiva, la resolución de problemas involucra la valoración de posibilidades y la toma de decisiones acertadas según el contexto lo demande, lo que convierte al aprendizaje-servicio en una metodología compleja.

\section{B. Reconocimiento institucional}

En el entendido que el reconocimiento institucional se entenderá como aquellas declaraciones, directas o indirectas, que la institución realiza sobre el aprendizaje-servicio, los participantes valoran que el modelo educativo haga mención explicita a la importancia de la metodología de aprendizaje-servicio:

\footnotetext{
"Aunque si hay que reconocer que [está] en instrumentos como el Modelo Educativo Institucional, que esta puesto en los procesos de acreditación de la universidad, en la página web, en todos lados, uno podría entender que eso es un aspecto incipiente, prematuro de institucionalización". (Grupo focal, 17 de agosto 2016)

Dicho reconocimiento es importante, pero no suficiente a ojos de los participantes. Pareciera ser que la declaración no alcanza a cubrir aspectos de implementación, como se
} 
señala a continuación:

"[Existe] un vacío en la bajada de la política universitaria" (Grupo focal, 17 de agosto 2016)

"La bajada -a lo que podríamos llamar política universitaria y gestión- es el déficit que tenemos". (Grupo focal, 17 de agosto 2016)

"Hay elementos de institucionalización, eso es así. Lo que no es lo mismo que entender la institucionalización como la promoción a través de instrumentos de gestión administrativa". (Grupo focal, 17 de agosto 2016)

De hecho, no existe mención a que las declaraciones institucionales se grafiquen en alguna política en particular, ya sea a nivel curricular o de recursos. Por el contrario, parecen demandar una atención más directa y manifiesta:

"Distinto es que en el marco de ciertos fondos que tiene la universidad, uno puede apoyarse en ellos. No es que exista un apoyo al aprendizaje-servicio, lo que existe son instancias, fondos, unidades de la universidad a los cual uno puede postular y en ellos se puede apoyar". (Grupo focal, 17 de agosto 2016)

Lo anterior advierte que desde la visión de los docentes se identifican acciones de reconocimiento mediante el fomento a la innovación en general, pero no como un apoyo dirigido en particular al aprendizaje-servicio.

\section{Integración curricular}

Los procesos de renovación curricular en el interior de la institución son algo relativamente nuevo, sin embargo los docentes reconocen que pueden ser instancias para formalizar el aprendizaje-servicio:

"Y a la vez, la [unidad de apoyo a la docencia] tiene toda la área de lo que es la reestructuración de las malla curriculares, o sea, trabaja en conjunto con eso. ¿Por qué no es posible instalarlo dentro de la malla?

". (Grupo focal, 17 de agosto 2016)

"Perfectamente podría -yo cuando pienso en esta cuestión en grande y fantaseo digamos- me gusta el modelo de la [referencia a una universidad chilena], ahí todas las carreras deben tener experiencia en aprendizaje servicio, y los estudiantes no pueden pasar por alto" (Grupo focal, 17 de agosto 2016)

De lo anterior se puede inferir que para algunos docentes la respuesta sobre institucionalización estaría en la obligatoriedad de incluir el aprendizajeservicio en todos los planes de estudio. Sin embargo, la institución no posee lineamientos para forzar ningún tipo de metodología al interior de las carreras, sino más bien, lo que se declara es la promoción de las mismas y su adopción, en función del perfil de egreso profesional de cada una de ellas.

No está claro si la demanda nace por el desconocimiento de los lineamientos institucionales que son flexibles respecto de la innovación educativa, o bien, un deseo de formalizar y universalizar las iniciativas de aprendizaje-servicio.

D. Soporte institucional

Con respecto al apoyo material, los docentes destacan la importancia de contar con recursos materiales para el 
desarrollo de las actividades que se enmarcan en aprendizaje-servicio, recursos que permitan apoyar las labores docentes y posibiliten a los profesores contar con tiempo para retroalimentar a los estudiantes, apoyar el trabajo en las comunidades, planificar y diseñar sus actividades.

Por ello, se valora ampliamente la existencia de los proyectos de innovación docente que proveen financiamiento para la mejora educativa en diversas áreas. Sin embargo, acusan restricciones en el uso de los gastos, como se señala a continuación:

"Yo encuentro que el proyecto efectivamente nos apoyó en el equipamiento, porque gracias al proyecto pudimos tener un computador, impresora con fotocopiadora que ha servido mucho para la comunidad. $Y$ también se usaron recursos para que con una colega fuéramos a presentar el trabajo a Argentina. Pero hay otras cosas que no nos permitían en los gastos". (Grupo focal, 17 de agosto 2016)

No obstante, se hace notar que al no existir un apoyo específico para el aprendizaje-servicio no se considera la especificidad de los gastos de esta metodología, como transporte de los estudiantes, materiales y alimentación:

"Dentro de la evaluación de la asignatura, lo primero que salió, que está asignatura era enferma de cara, nosotros les hacíamos más encima gastar plata en preparar materiales -Los modelos de goma eva, con cartulinas, con distintos materiales de escritorio- que nadie se las pagaba" (Grupo focal, 17 de agosto 2016).

"Entonces se creó la necesidad de que los chiquillos necesitaban comer algo antes de empezar la intervención, que empieza a las
2 de la tarde en el colegio" (Grupo focal, 17 de agosto 2016).

En relación al apoyo técnico -como asesores- y administrativo, se reconoce y valora la existencia de una unidad de apoyo docencia:

"La aplicación no se ha institucionalizado encuentro yo... aparte de lo que ustedes hacen [unidad de apoyo]". (Grupo focal, 17 de agosto 2016)

Se reconocen a su vez, la realización de cursos y talleres sobre aprendizajeservicio, lo que les permite capacitarse permanentemente:

"Hemos recibido apoyo dentro de los PID, pero además de la capacitación que hace [la unidad de apoyo a la docencia] como formular programas, de asignatura, que sé yo, metodologías activas de enseñanza" (Grupo focal, 17 de agosto 2016).

Considerando que dicha unidad de apoyo a la docencia, no contempla acciones administrativas ni de gestión, elemento que los profesores evidencian deficiente al interior de la universidad, sobre todo en la relación con los socios comunitarios:

"[existe] un nivel superficial, temprano de institucionalización y que, para llegar a un nivel mayor de madurez [requiere] la concreción de convenios con socios comunitarios" (Grupo focal, 17 de agosto 2016)

"Creo que la intención puede estar, pero yo creo que falta todavía mucho camino por recorrer, bajar esa intención a lo más concreto e ir formalizando este tipo de relaciones [con socios comunitarios]" (Grupo focal, 17 de agosto 2016) 
Este punto resulta de gran importancia pues la literatura ya da cuenta de la importancia del socio comunitario como un agente clave en las iniciativas de aprendizaje-servicio, así como la necesidad de poseer convenios permanentes en el tiempo que, además de facilitar la ejecución de los proyectos, implique la creación de lazos de confianza y cooperación entre todos los actores involucrados.

Finalmente, se señala una problemática de gran envergadura como es el tipo de contrato y jornada que los profesores universitarios poseen. Si bien, es posible que ella exceda a la situación del aprendizaje-servicio, no es menos cierto que la metodología tiene ciertos requerimientos que otras asignaturas no tienen, como se señala a continuación:

\footnotetext{
"Hay muchísimos profesores por hora que hacen aprendizaje-servicio. Me saco el sombrero porque francamente no les da tiempo para nada, les da tiempo para estar directamente con el alumno y punto, nada más; no le da tiempo ni para preparar sus clases. Además, les pagan menos que a los profesores que están contratados por jerarquía académica" (Grupo focal, 17 de agosto 2016)
}

Esta tensión para la institucionalización resulta relevante en la medida que viene a reforzar lo planteado en la literatura respecto de que gran parte de las iniciativas de aprendizaje-servicio se sostienen en esfuerzos individuales, que lamentablemente, pueden desaparecer rápidamente en el tiempo.

\section{Conclusiones}

El análisis presentado da cuenta de la visión que posee un grupo de docentes universitarios sobre los procesos de institucionalización del aprendizajeservicio desarrollados en una institución de Educación Superior de carácter estatal chilena.

En primer lugar, podemos señalar que todos los participantes reconocen en el aprendizaje-servicio su capacidad para desarrollar aprendizajes en la línea de la resolución de problemas. No obstante, las potencialidades que esta metodología tiene para desarrollar una competencia reflexiva, ciudadana y de responsabilidad social, son levemente declaradas. Lo anterior nos llama a atender las maneras en que el reconocimiento de la metodología a tomado forma a nivel institucional.

Este punto resulta de especial relevancia si consideramos que es el reconocimiento institucional el que dará las claves y orientaciones sobre qué es el aprendizaje-servicio, su rol y su relevancia para el cumplimento de la misión de la universidad. De hecho, los participantes identifican el aprendizajeservicio en las declaraciones institucionales, pero lo ven poco concreto y dentro de una serie de otras iniciativas muy diferentes. Al no contar con un reconocimiento más específico, existe el riesgo de diluir las experiencias existentes, restándole continuidad e identidad en el tiempo.

Por otra parte, no existe una posición única respecto de cómo podrían materializarse las políticas institucionales hacia el aprendizajeservicio. A nivel microcurricular, todos los participantes cuentan con garantías de su implementación en el programa 
de asignatura, pero reconocen la inestabilidad en tanto solo depende de ellos la continuidad de la experiencia de aprendizaje-servicio. Por otra parte, sí saben que es posible que se instale directamente en los planes de estudio, pero dejan entrever cierta falta de apoyo a nivel político.

Desde el punto de vista de los soportes institucionales, los participantes reconocen las acciones de capacitación docente y el financiamiento directo a proyecto de innovación. No obstante, este último podría necesitar recoger las especificidades que los proyectos de aprendizaje-servicio tienen y que los procedimientos generales no logran cubrir. Por ejemplo, el gasto de recursos suele estar asociado a compra de libros, materiales de laboratorio y pago de estudiantes ayudantes; sin embargo no contempla formas de rendir gastos asociados a desplazamiento y transporte, alimentación para los estudiantes y el socio comunitario, por nombrar algunas.

Es importante destacar, que algunos de los problemas que los participantes observan en la implementación del aprendizaje-servicio exceden al desarrollo de la metodología y responden a problemas estructurales en el sistema de educación superior chileno, como es el financiamiento a profesores de tiempo parcial.

Reconociendo esto último, si podemos señalar que la metodología de aprendizaje-servicio tiene requerimientos específicos de tiempo por parte del docente y de los estudiantes, por lo que al menos debiese contar con una carga académica diferenciada de otras asignaturas.

De lo anterior, se evidencian ciertos elementos de avance en la formalización del aprendizaje-servicio, lo que se podría denominar institucionalización temprana, caracterizada por la existencia de ciertos apoyos institucionales, entre los que destacan el apoyo financiero y las instancias de formación docente en apoyo a la metodología. No obstante, existe un nudo crítico importante, que se relaciona principalmente con la integración estructural y procedimental del aprendizaje-servicio (Curry, 1992). Este elemento, permitiría la definición de una estructura o entidad institucional que gestione las diversas tareas, articule todos los apoyos dispersos en la institución y defina/implemente procedimientos que permitan dar sustentabilidad a las experiencias (Jouannet et al., 2015).

Por otra parte, al hablar de reconocimiento institucional se suele señalar la inequitativa relación entre las actividades de docencia e investigación, siendo estas últimas mucho mejor ponderadas en los procesos de evaluación del desempeño. Si bien esta tensión afecta a gran parte de las organizaciones de educación superior del mundo, no es menos cierto que una forma de reconocer institucionalmente las actividades de docencia y en particular el aprendizaje-servicio, es promoviendo el estudio sistemático de las innovaciones, que permitan evaluar los resultados obtenidos en formación ciudadana y responsabilidad social.

Finalmente, consideramos la pauta para

Almendras-Chacana, R. y Pérez-Lorca, A. (2019). Percepciones docentes en torno a los procesos de institucionalización del aprendizaje-servicio en educación superior: un estudio de caso. RIDAS, Revista Iberoamericana de Aprendizaje Servicio, 7, 17-36. DOI10.1344/RIDAS2019.7.2 
el desarrollo del grupo focal pudo constituir una limitante para indagar en mayor profundidad sobre la identidad y concepciones del profesorado en relación al aprendizaje-servicio. En este sentido, creemos puede ser una interesante línea de desarrollo posterior, lo que podría proporcionar orientaciones respecto de cómo fortalecer el reconocimiento de la metodología y sus participantes a nivel institucional.

\section{Referencias bibliográficas}

Agrafojo, J., García-Antelo, B. y Jato, E. (2017). Aprendizaje servicio e innovación educativa en la Universidad de Santiago de Compostela: estrategia para su institucionalización. RIDAS, Revista Iberoamericana de Aprendizaje Servicio, 3, 23-34.

Biggs, J. (2006). Calidad del aprendizaje universitario. Madrid: Narcea.

Bringle, R. y Hatcher, J. (1996). Implementing Service Learning in Higher Education. The Journal of Higher Education, 67(2), 221-239.

Bringle, R. y Hatcher, J. (2000). Institutionalization of Service Learning in Higher Education. The Journal of Higher Education, 71(3), 273-290.

Campo, L. (2015). Evaluar para mejorar los proyectos de aprendizaje servicio en la universidad. RIDAS, Revista Iberoamericana de Aprendizaje y Servicio, 1, 91-111.

Canales, M. (2006). Metodologías de la investigación social. Introducción a los oficios. Santiago: LOM.

Carr, W. (1996). Una teoría para la educación. Hacia una investigación educativa crítica. Madrid: Morata.

Coll, C. (2001). Constructivismo y educación: la concepción constructivista de la enseñanza y del aprendizaje. En C. Coll, J. Palacios y A. Marchesi (comps.) Desarrollo Psicológico y Educación. Vol 2. Psicología de la Educación Escolar (pp. 157-186). Madrid: Alianza Editorial.

Coll, C., Colomina, R., Onrubia, J., y Rochera, M. J. (1992). Actividad conjunta y habla: una aproximación al estudio de los mecanismos de influencia educativa. Infancia y Aprendizaje, 59$60,189-232$.

Curry, B. K. (1992). Instituting Enduring Innovations: Achieving Continuity of Change in Higher Education. ASHE-ERIC Higher Education Report, 7.

Furco, A. (1999). Research Notes. National Society for Experiential Education Quarterly.

Furco, A. (2001). Advancing ServiceLearning at Research Universities. New Directions for Higher Education, 114, 67-78.

Furco, A. (2011). El aprendizajeservicio: un enfoque equilibrado de la educación experiencial. Revista Educación Global, 64-70.

Geruzaga, M. y García, A. (2018). ABProblemas, ABProyectos, ABRetos...¿¿Legado de Dewey? Valor 
añadido del ApS respecto a "otros" aprendizajes experienciales. En El Aprendizaje y Servicio en la Universidad. Una metodología docente y de investigación al servicio de la justicia social y desarrollo sostenible (pp. 22-27). Salamanca: Comunicación Social.

Gezuraga, M. y Herrero, M. A. (2017). El aprendizaje servicio en universidades de Buenos Aires: avanzando hacia su institucionalización. RIDAS, Revista Iberoamericana de Aprendizaje Servicio, 3, 4-22.

Hernández, R., Fernández, C. y Baptista, P. (2010). Metodología de la investigación. Mexico D.F: Mc Graw Hill.

Jouannet, C., Ponce, C., Montalva, J. T. y Von Borries, V. (2015). Diseño de un modelo de institucionalización de la metodología de aprendizaje servicio en educación superior Palabras clave. RIDAS. Revista Iberoamericana de Aprendizaje Servicio, 1, 112-131.

Malpica, F. (2013). Calidad de la práctica educativa. Barcelona: GRAO.

Marchant, P. y Foster, C. (2017). Una metodología de análisis de casos para orientar la toma de decisiones en el contexto escolar. En El poder de la evaluación en el aula (pp. 301-339). Santiago: Ediciones UC.

Martínez, B., Martínez, I., Alonso, I. y Gezuraga, M. (2013). El aprendizajeservicio, una oportunidad para avanzar en la innovación educativa dentro de la Universidad del País Vasco. Tendencias Pedagógicas, 21, 99-117.
Martínez, M. (2010). Aprendizaje servicio y responsabilidad social de las universidades. Barcelona:

Octaedro/ICE.

Martínez, V., Medero, N., Ibáñez, E. y Sánchez, M. (2018). El Aprendizaje Servicio en la Universidad. Una metodología docente y de investigación al servicio de la justicia social y desarrollo sostenible. Salamanca: Comunicación Social.

Martínez-Usarralde, M., Zayas, B., y Sahuquillo, P. (2016). Aps y ciudadanía comprometida: aproximación descriptiva-exploratoria a un caso. Opción, 32(8), 187-208.

Mauri, T., Coll C. y Onrubia, J. (2007). La evaluación de la calidad de los procesos de innovación docente universitaria. Una perspectiva constructivista, 1-11. Recuperado de http://diposit.ub.edu/dspace/handle/24 $45 / 21983$

Mella, O. (2010) Grupos focales. Técnica de investigación. Documento de trabajo no 3. Santiago: CIDE.

Páez, M. y Puig, J. (2013). La reflexión en el Aprendizaje Servicio. Revista Internacional de Educación para la Justicia Social, 2(2), 13-32.

Pérez, G. (2007). Investigación cualitativa. Retos e interrogantes. Madrid: La Muralla.

Phillips, A., Bolduc, S. R. y Gallo, M. (2013). Curricular Placement of Academic Service-Learning in Higher Education. Journal of Higher Education Outreach and Engagement, 17(4), 75- 
96.

Puig, J., Gijón, M., Martín, X. y Rubio, L. (2011). Aprendizaje-servicio y Educación para la Ciudadanía. Revista de Educación, Número Ext, 45-67.

Rodríguez, D. y Valldeoriola, J. (2009). Metodología de la investigación.

Barcelona: Universitat Oberta de Catalunya (UOC).

Romero, J. (2003). Innovación en la enseñanza universitaria. Revista de Educación, 7, 113-136.

Ruiz, M., Meneses, A. y Montenegro, M. (2014). Coherencia curricular y oportunidades para aprender Ciencias. Ciencia y Educação, 20(4), 955-970.

Santos, M.; Sotelino, A. y Lorenzo, M. (2015). Aprendizaje-servicio y misión cívica de la universidad. Una propuesta de desarrollo. Barcelona: Octaedro.

Stake, R. (1998). Investigación con estudio de casos. Madrid: Ediciones Morata.

Stater, K. J. y Fotheringham, E. (2009). Mechanisms for Institutionalizing Service-Learning and Community Partner Outcomes. Journal of Higher Education Outreach and Engagement, 13(2), 7-30.

Tapia, N. (2010). La propuesta pedagógica del "aprendizaje-servicio": una perspectiva latinoamericana. Tzhoecoen, 5, 23-43.

Tapia, N. (2016). Inserción curricular del aprendizaje-servicio en la Educación Superior. Buenos Aires: CLAYSS.
UNESCO (2015) Declaración de Incheon. Educación 2030. Recuperado de

http://unesdoc.unesco.org/images/002 3/002338/233813M.pdf

UNESCO (2017) Educación para los Objetivos de Desarrollo Sostenible: objetivos de aprendizaje. Recuperado de https://unesdoc.unesco.org/ark:/48223 /pf0000252423

Almendras-Chacana, R. y Pérez-Lorca, A. (2019). Percepciones docentes en torno a los procesos de institucionalización del aprendizaje-servicio en educación superior: un estudio de caso. RIDAS, Revista Iberoamericana de Aprendizaje Servicio, 7, 17-36. DOI10.1344/RIDAS2019.7.2 